\title{
Seroprevalence and risk factors of Kaposi's sarcoma-associated herpesvirus infection among the general Uygur population from south and north region of Xinjiang, China
}

\author{
Hui Wang ${ }^{1}$, Jian Liu', Dilimulati ${ }^{1}$, Liang Li ${ }^{1}$, Zhihui Ren ${ }^{1}$, Hao Wen ${ }^{1}$ and Xing Wang ${ }^{1,2^{*}}$
}

\begin{abstract}
Background: Kaposi sarcoma (KS) is a complex multifocal neoplasm and is the major cause of death for about $50 \%$ of acquired immunodeficiency syndrome (AIDS) patients. Kaposi's sarcoma-associated herpesvirus (KSHV) is an oncogenic virus with a causal role in the development of all types of KS. KS is prevalent among the Uygur people in Xinjiang, especially in south area. Here we carried out a cross-sectional study among 1534 general Uygur individuals from south and north region of Xinjiang to assess the seroprevalence of KSHV and to identify the potential correlation between KSHV seroprevalence and KS incidence.

Results: Seroprevalence of KSHV in South and North Xinjiang was 23.1\% and 25.9\%, respectively. Older age was independently associated with higher KSHV seroprevalence. In subjects from South Xinjiang, lower educational level and reported drinking were each independently associated with higher KSHV seroprevalence. Furthermore, the antibody titer was significantly lower in both south and north KSHV seropositive individuals compared with KS patients, as analyzed by gradient dilution $(P<0.001)$.

Conclusion: KSHV is highly prevalent in the general Uygur population in both South and North Xinjiang. Interestingly, the infection rate of KSHV in these two geographical areas did not correlate well with KS incidence. Perhaps unknown factors exist that promote the progression of KSHV infection to KS development in the local minority groups.
\end{abstract}

Keywords: Kaposi's sarcoma-associated herpesvirus (KSHV), Kaposi's sarcoma (KS), seroprevalence, Uygur people, risk factors, Xinjiang

\section{Background}

Kaposi sarcoma (KS) is a mesenchymal tumour involving blood and lymphatic vessels [1]. KS can be classified according to its clinical and epidemiological characteristics and the different types include: classical, acquired immunodeficiency syndrome (AIDS)-related, iatrogenic and endemic KS $[2,3]$. Notably, KS is the most common cancer associated with AIDS worldwide [4]. Approximately $20 \%$ of AIDS patients develop KS in Western countries and AIDS-KS is the major cause of death for

\footnotetext{
* Correspondence: xwang@sibs.ac.cn

${ }^{1}$ The First Teaching Hospital of Xinjiang Medical University,1 Liyu Shan Road, Urumqi, Xinjiang, People's Republic of China

Full list of author information is available at the end of the article
}

about $50 \%$ of AIDS patients [5,6]. Kaposi's sarcoma-associated herpesvirus (KSHV) also known as Human herpesvirus 8 (HHV-8), is an oncogenic virus with a causal role in the development of KS [2,7-9], and two other AIDSrelated lymphoproliferative disorders: primary effusion lymphoma (PEL) and the plasma-cell variant of multicentric Castleman's disease (MCD) [10]. KSHV has been detected in the lesions of nearly all patients with KS $[11,12]$, and when detected in blood it is predictive of the development of KS $[8,13]$.

KSHV prevalence exhibits considerable variation in different geographical regions and populations. Several studies have demonstrated that KSHV seroprevalence correlates with the occurrence of KS [14-17]. In most

\section{Biomed Central}


Asian countries, the seroprevalence of KSHV ranges from $0 \%$ to $3 \%$, which is consistent with a generally lower incidence of KS in this region [18]. In most provinces of China, KSHV seroprevalence was less than $8 \%[19,20]$. However, the Xinjiang area, located northwest of China, exhibited a distinct pattern. Over $95 \%$ of KS cases in China occurred in Xinjiang, especially classic cases of KS which predominantly occurred in minority groups, particularly in older men [21]. Recent studies have found KSHV seroprevalence correlates with the high incidence of KS in Xinjiang, which ranged from $12.5 \%$ to $48.0 \%$ in different study populations, including the general population, blood donors, tumor patients and HIV-infected individuals [18,21-24]. The incidence of HIV infection has increased rapidly in Xinjiang over the past few years. Thus, increasing numbers of AIDS-KS cases have recently been reported in this area. It is therefore of great medical importance to investigate KSHV seroprevalence and transmission mode-associated behaviors in Xinjiang, to gain a greater epidemiological understanding of these diseases, and to then be able to apply these findings to improve public health strategies.

Xinjiang is located at the middle point of the Silk Road that used to extend from Rome to China. Many ethnicities, such as the Uygur (48\%), Han (38\%) and Kazakh people (7\%), mix in this area. Classical KS cases occur most frequently in two of these minority groups: the Uygur and Kazakh groups. Furthermore, about $90 \%$ of KS cases have been reported in the Uygur group, which reside in the south region of Xinjiang [21]. However, the limitations of previous studies have been that they were generally either restricted to Uygur patients or mainly from the northern part of Xinjiang [18,21,22], where the socio-economic status is higher than in other areas. The objective of the current study was to investigate the potential correlation between KSHV seroprevalence and KS incidence in Xinjiang endemic areas, and to determine whether environmental or sanitation-associated issues effect KSHV infection rates or KS incidence in this ethnic background.

\section{Results}

Characteristics of the study population and KSHV seroprevalence

Of the 1534 Uygur people included in the study, 1008 (65.7\%) were from the southern part of Xinjiang and 526 (34.3\%) were from the northern part of Xinjiang. The seroprevalence of KSHV among the general Uygur population in the southern and northern parts of Xinjiang was $23.1 \%$ and $25.9 \%$, respectively. Table 1 presents the associations with gender, age and education stratified by region (Southern and Northern). The lower education associated with KSHV seroprevalence only in south region of Xinjiang.

A multivariate logistic regression analysis was used to identify independent risk factors for KSHV infection in total study population (Table 2). In this model, KSHV was significantly more prevalent in individuals aged $\geq 55$ years compared to those aged $<55$ years. Moreover, place of residence was also independently associated with KSHV seroprevalence.

\section{KSHV seropositivity among 1008 subjects from south region of Xinjiang}

The characteristics of the 1008 Uygur village participants from Southern Xinjiang and the KSHV seroprevalence rates are presented in Table 3. In this study population, 497 subjects were male $(49.3 \%)$ and 511 were female (50.7\%). The age distribution was from 18 to 91 years old. Among 39 subjects positive for hepatitis $B$ and $C$ viruses (HBV and $\mathrm{HCV}), 11$ were KSHV positive (28.2\%). Interestingly, participants who were regular drinkers exhibited high serum prevalence of KSHV (46.2\%). Table 3 illustrates the univariate associations between KSHV infection and the 1008 participants characteristics. KSHV infection was associated with advancing age $(P=0.020)$. More

Table 1 The associations of KSHV seroprevalence with gender, age and education stratified by geographic region (Southern and Northern) in general Uygur subjects from Xinjiang, China

\begin{tabular}{|c|c|c|c|c|c|c|c|c|}
\hline \multirow[t]{2}{*}{ Characteristic } & \multicolumn{4}{|c|}{ Moyu country(Southern) } & \multicolumn{4}{|c|}{ Urumqi(Northern) } \\
\hline & No.of Subjects & KSHV seropositivity $\mathrm{n}(\%)$ & $\chi^{2}$ & $P$ & No.of Subjects & KSHV seropositivity $\mathrm{n}(\%)$ & $\chi^{2}$ & $P$ \\
\hline Gender & & & 0.337 & 0.562 & & & 0.411 & 0.521 \\
\hline Male & 497 & $111(22.3)$ & & & 287 & $71(24.7)$ & & \\
\hline Female & 511 & $122(23.8)$ & & & 239 & $65(27.2)$ & & \\
\hline Age (years) & & & 2.768 & 0.096 & & & 0.446 & 0.504 \\
\hline$<40$ & 584 & $124(21.2)$ & & & 480 & $126(26.3)$ & & \\
\hline$\geq 40$ & 424 & 109(25.7) & & & 46 & $10(21.7)$ & & \\
\hline Education (years) & & & 6.632 & 0.010 & & & 2.340 & 0.126 \\
\hline$\leq 6$ & 720 & $182(25.3)$ & & & 24 & $3(12.5)$ & & \\
\hline$>6$ & 288 & $51(17.7)$ & & & 502 & 133(26.5) & & \\
\hline Total & 1088 & 233(23.1) & & & 526 & $136(25.9)$ & & \\
\hline
\end{tabular}


Table 2 Multivariate logistic regression analysis: determinants of KSHV seropositivity

\begin{tabular}{cccc}
\hline Characteristic & OR & $\mathbf{9 5 \% ~ C l}$ & $\boldsymbol{P}$ value \\
\hline $\begin{array}{c}\text { Gender } \\
\text { Male }\end{array}$ & 1.00 & & \\
Female & 1.08 & $0.86-1.37$ & 0.51 \\
Age & & & \\
$<40$ & 0.69 & $0.47-1.03$ & 0.07 \\
$40-55$ & 0.63 & $0.41-0.96$ & 0.03 \\
$\geq 55$ & 1.00 & & \\
Education level & & & \\
Illiterate/semiliterate & 1.44 & $0.89-2.30$ & 0.13 \\
Elementary school & 1.26 & $0.90-1.76$ & 0.18 \\
Junior high school & 1.00 & & \\
region & & & \\
Moyu country & 1.00 & & \\
Urumqi & 1.49 & $1.07-2.08$ & \\
\hline
\end{tabular}

specifically, KSHV seroprevalence increased with age from $21.2 \%$ in those aged $<40$ years to $21.3 \%$ in those aged $40-55$, and to $30.7 \%$ in those aged $\geq 55$ years. Furthermore, there was a significant difference in KSHV seroprevalence with respect to educational level $(\mathrm{P}=0.020)$, from $17.7 \%$ in those educated to junior high school level, to $24.3 \%$ in those educated to elementary school level, and to $28.3 \%$ in those who were illiterate/semiliterate. An association of borderline significance was detected between KSHV seropositivity and the report of drinking $(\mathrm{P}=0.058)$. No associations were observed between KSHV infection and gender, family number, BMI and HBV or HCV infection.

To further identify independent risk factors, variables that were significant in univariate analysis at $\mathrm{P}=0.100$ were included in multivariate logistic regression analysis and the data are presented in Table 4 . Lower educational level (Illiterate/semiliterate vs. Junior high school, OR = $1.67,95 \% \mathrm{CI}=1.02-2.73$ ) and reported drinking (yes vs.

Table 3 Univariate analysis of KSHV seroprevalence in the subjects from southern part of Xinjiang

\begin{tabular}{|c|c|c|c|c|c|}
\hline Characteristic & Uygur people $n(\%)$ & KSHV seropositivity $\mathrm{n}(\%)$ & OR & $95 \% \mathrm{Cl}$ & $P$ value \\
\hline \multicolumn{6}{|l|}{ Gender } \\
\hline Male & 497 & $111(22.3)$ & 1.00 & & \\
\hline Female & 511 & $122(23.8)$ & 1.10 & $0.81-1.46$ & 0.56 \\
\hline \multicolumn{6}{|l|}{ Age } \\
\hline$<40$ & 584 & $124(21.2)$ & 1.00 & & \\
\hline $40-55$ & 225 & $48(21.3)$ & 1.01 & $0.69-1.47$ & 0.98 \\
\hline$\geq 55$ & 199 & $61(30.7)$ & 1.64 & $1.14-2.35$ & 0.01 \\
\hline \multicolumn{6}{|l|}{ Education level } \\
\hline Illiterate/semiliterate & 180 & $51(28.3)$ & 1.00 & & \\
\hline Elementary school & 540 & $131(24.3)$ & 0.81 & $0.55-1.18$ & 0.28 \\
\hline Junior high school & 288 & $51(17.7)$ & 0.54 & $0.35-0.85$ & 0.01 \\
\hline \multicolumn{6}{|l|}{ Family number } \\
\hline$\leq 5$ people & 641 & $151(23.6)$ & 1.00 & & \\
\hline$>5$ people & 367 & $82(22.3)$ & 0.93 & $0.69-1.27$ & 0.66 \\
\hline \multicolumn{6}{|l|}{ Smoking } \\
\hline No & 931 & $217(23.3)$ & 1.00 & & \\
\hline Yes & 77 & $16(20.8)$ & 0.86 & $0.49-1.53$ & 0.61 \\
\hline \multicolumn{6}{|l|}{ Drinking } \\
\hline No & 995 & $227(22.8)$ & 1.00 & & \\
\hline Yes & 13 & $6(46.2)$ & 2.90 & $0.97-8.72$ & 0.06 \\
\hline \multicolumn{6}{|l|}{ HBV } \\
\hline Negative & 983 & $226(23.0)$ & 1.00 & & \\
\hline Positive & 25 & 7 (28.0) & 1.30 & $0.54-3.16$ & 0.56 \\
\hline \multicolumn{6}{|l|}{$\mathrm{HCV}$} \\
\hline Negative & 994 & $229(23.0)$ & 1.00 & & \\
\hline Positive & 14 & 4 (28.6) & 1.34 & $0.42-4.30$ & 0.63 \\
\hline \multicolumn{6}{|l|}{ BMI } \\
\hline$\geq 25$ & 330 & $73(22.1)$ & 1.00 & & \\
\hline$<25$ & 678 & $160(23.6)$ & 0.92 & $0.67-1.26$ & 0.60 \\
\hline Total & 1008 & $233(23.1)$ & & & \\
\hline
\end{tabular}


Table $\mathbf{4}$ Independent risk factors for KSHV infection in southern part of Xinjiang

\begin{tabular}{llll}
\hline Characteristic & OR & $\mathbf{9 5 \% ~ C l}$ & $P$ value \\
\hline $\begin{array}{l}\text { Age (years) } \\
\quad<40\end{array}$ & 1.00 & & \\
$\quad 40-55$ & 0.91 & $0.62-1.35$ & 0.64 \\
$\quad \geq 55$ & 1.42 & $0.95-2.11$ & 0.08 \\
$\quad \begin{array}{l}\text { Education level } \\
\quad \text { Junior high school }\end{array}$ & 1.00 & & \\
$\quad$ Elementary school & 1.48 & $1.02-2.14$ & 0.04 \\
$\quad$ Iliterate/semiliterate & 1.67 & $1.02-2.73$ & 0.04 \\
Drinking & & & \\
$\quad$ No & 1.00 & & \\
$\quad$ Yes & 3.23 & $1.07-9.78$ & 0.04 \\
\hline
\end{tabular}

no, $\mathrm{OR}=3.23,95 \% \mathrm{CI}=1.07-9.78)$ were each independently associated with higher KSHV seroprevalence.

\section{Antibody titers of KSHV in highly seropositive individuals and KS patients}

The distribution pattern of KSHV antibody titers among high seropositive individuals from both north and south and KS patients were compared and the results are displayed in Figure 1. The individual number of low antibody titer group in south, north and KS patients was 35,40 and 28 . For medium group, the number is 5,0 and 11 . However, the number is 0,0 and 12 in south, north and KS patients for high $\operatorname{group}\left(\chi^{2}=33.74, \mathrm{P}<0.001\right)$. These results indicated that compared with $\mathrm{KS}$ patients, KSHVinfected individuals in both south and north presented a lower antibody titer against KSHV.

\section{Discussion}

More than $95 \%$ of KS cases in China, including all epidemiological forms, were observed in minority groups in Xinjiang, particularly in the Uygur group [21]. Previous studies have shown a high seroprevalence of KSHV in the range of $12.5 \%$ to $48.0 \%$ in different study populations in

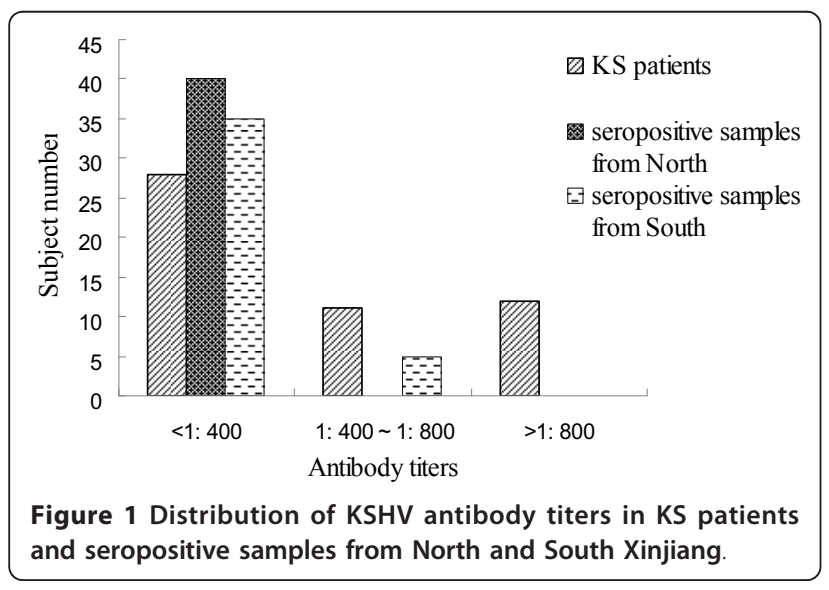

this region, that correlated well with a high incidence of KS [18,21-23]. However, these studies were mainly restricted to Uygur patients with diseases other than $\mathrm{KS}$, and the study populations were mainly from the northern part of Xinjiang $[18,21]$. Thus the discrepancy between the socio-economic status in these two geographical areas and the absence of behavior-associated data, mean that these studies did not take other variables into account. In the current cross-sectional study, the seroprevalence of KSHV among the general Uygur population in the southern and northern parts of Xinjiang was $23.1 \%$ and $25.9 \%$, respectively, which is in agreement with previous findings from Xinjiang [21-24]. There was no significant difference in KSHV infection between the two regions of Xinjiang, indicating that minority group background is still the most important factor in KSHV infection. Although as expected, titers were higher in $\mathrm{KS}$ cases than in the general population, titers in the general population did not differ by geography, unlike the incidence of KS which is much higher in the South. Combined with the distribution pattern of KSHV antibody titers among seropositive individuals and KS patients, our findings suggested that other unknown factors may exist in South Xinjiang which potentially promote the development from KSHV infecton during KS pathogenesis. To further confirm this hypothesis, a prospective study focused on geographical and ethnic parameters should be carried out.

The seroprevalence of KSHV in the general Uygur population in Xinjiang was significantly more prevalent in individuals aged $\geq 55$ years compared to those aged $<55$ years. These results are consistent with those of several other studies, which described an increase in seroprevalence of KSHV with age in different populations [25-29]. This correlation may be related to the natural aging process. Generally, the immunity of older people is weaker than that of younger people, and hypoimmunity maybe a promoting agent for KSHV infection. Moreover, it is notable that KSHV prevalence was similar in men and women which is consistent with many studies $[18,29]$. and our previous researches $[21,23]$ that find the distribution of KSHV seroprevalence has no difference by sex.

Further analyses were then carried out to focus on the 1008 rural Uygur people from South Xinjiang. This is the first report regarding KSHV seroprevalence and risk factors in this area, a region endemic for KS. The seroprevalence of KSHV in this study population was independently associated with educational level. It was apparent that with improved educational level (from illiterate/semiliterate to Junior high school level education), KSHV seroprevalence decreased from $28.3 \%$ to $17.7 \%$, suggesting that a higher education level is a protection factor for KSHV infection. Notably, the educational level of this study population was usually below the high school level, and among the older age group $(\geq 55)$ the percentage of illiterate/semiliterate 
individuals was $43.7 \%$. Therefore, knowledge about health issues is far more limited in the Uygur population in this region, especially in the elder Uygur population. Furthermore, the mobility of the population in this region is relatively low. The traditional life style and behavior of the Uygur people has remained unchanged over the past two decades [30], with the life quality and level of education reflecting their low socioeconomic status. Such conditions might facilitate the transmission of KSHV infection, in line with with previous reports linking KSHV to rural settings in Italy and Sicily [31-33]. Interestingly, we also identified drinking could increase the risk of KSHV infection, a result consistent with previous studies [34,35]. Among 13 drinkers, six were KSHV positive (46.2\%). Because of the small sample size, the relationship between drinking and KSHV infection requires further investigation in a larger sample population in Xinjiang.

\section{Conclusions}

We identified a high seroprevalence of KSHV infection in the general Uygur population in both South and North Xinjiang, and the distribution of KSHV seroprevalence in these two geographical areas did not show a clear correlation with KS incidence. Perhaps other unknown factors have promoted the association between KSHV infection and KS development in the local minority groups. Understanding the epidemiology of KSHV infection in rural areas of Uygur is critical for designing effective intervention strategies to decrease the transmission of this virus and to prevent malignancies associated with KSHV infection.

\section{Methods \\ Study participants}

South Xinjiang was represented by Moyu Country of Hotan City which is located in the southernmost region of Xinjiang surrounding the Tarim Basin and where more than $95 \%$ of the population were rural Uygur people. North Xinjiang was represented by Urumqi, which is the most highly developed and industrialized region in Xinjiang. A total of 1543 research subjects were randomly selected from this two regions of Xinjiang by using a stratified, multistage sampling. Among them, residents (age > 18years) from 15 villages in 3 rural town of Moyu Country and employees from top 10 developed enterprises of Urumqi were 1008 and 526, respectively. Trained staff obtained the informed consent of all participants and carried out interviews. A questionnaire on age, gender, ethnicity, education level and residence was completed for all subjects. Other characteristics, such as family number, drinking, cigarette smoking and anthropometric measurements, including height, weight, waistline and hip circumference, were also recorded for all of the subjects in the southern part of Xinjiang. All data were analyzed anonymously. Body mass index (BMI) $\left(\mathrm{kg} / \mathrm{m}^{2}\right)$ values were also calculated for all subjects. Serum and plasma samples were collected, separated immediately and stored at $-80^{\circ} \mathrm{C}$ prior to testing.

Sera were also collected from samples taken from 51 patients with classic KS registered during the period between 1986 and 2008, at the First Teaching Hospital of Xinjiang Medical University in Urumqi (age: 17-83 years, 45 male vs. 6 female, 46 Uygur vs. 5 Kazakh, 46 from south region vs. 5 from other region of xinjiang), to compare antibody titers with KSHV infected persons. The study protocol was approved by the local research ethics committee of the First Teaching Hospital of Xinjiang Medical University. Written informed consents were obtained from all study subjects before data collection.

\section{Serological tests for KSHV}

Three KSHV specific viral genes were expressed and purified as GST-fusion proteins used to construct the combined antigens enzyme-linked immunosorbent assay (ELISA) including latent antigen ORF73 and lytic antigens ORF K8.1 and ORF65. Briefly, Viral antigens were diluted in coating buffer to a final concentration of $10 \mu \mathrm{g} / \mathrm{ml}$ and used to coat ELISA plates (Corning Glass Works, Corning, NY, USA) overnight at $4^{\circ} \mathrm{C}$. After washing with phosphatebuffered saline (PBS) containing 0.05\% Tween 20(PBST), 1:100 diluted serum samples were added and plates were incubated for $60 \mathrm{~min}$ at $37^{\circ} \mathrm{C}$. This was followed by incubation with goat anti-human IgG conjugated with peroxidase (Product No. A8667, Sigma, St. Louis, MO, USA) for $60 \mathrm{~min}$ at $37^{\circ} \mathrm{C}$. The color reaction was developed for 15 min at RT with tetramethyl benzidine after PBST washing. Reactions were stopped with $50 \mu \mathrm{l}$ of $1 \mathrm{M} \mathrm{H}_{2} \mathrm{SO}_{4}$ and absorbance was measured at a wavelength of $450 \mathrm{~nm}$. Serum samples from patients with classical-KS in Xinjiang and with AIDS-KS or skin carcinoma in France which were obtained from the Medical center of Besancon University France-Comte were used to assay the sensitivity and specificity in this study. Based on the surveys of above groups, the combined antigens ELISA had a sensitivity of $81.8 \%$ and specificity of $97.9 \%$, respectively. In the following screening, serum from an AIDS-KS patient that had high antibody titers to both KSHV latent and lytic antigens, and serum from three healthy children in Sichuan province without any specific antibodies to KSHV, were used as positive and negative controls, respectively, in all assays. Both positive and negative controls were used in three wells in each plate in this study. Each sample was also tested three times. Based on the results of assays with the control groups, a serum sample with an absorbance value above the mean plus three standard deviations of the negative control wells in an assay was considered as positive. A highly seropositive result for KSHV was set considered as a value greater than the mean absorbance value 
plus five standard deviations of the negative control wells. All assays were examined by a single observer.

Serum samples that were highly positive for antibodies against KSHV in the ELISA assay and the sera of $51 \mathrm{KS}$ patients were analyzed to determine their antibodiy titers using gradient serum dilutions (1:100,1:200,1:400,1:800, 1:1600 and 1:3200). The positive standard followed the criterion above. Antibody titers lower than 1:400, between 1:400 1:800 and higher than 1:800 were classified into the low group, medium and high group, respectively.

\section{Statistical analysis}

The data were entered in duplicate and analyzed using the SPSS11.0 software (SPSS, Chicago, IL).Univariate analysis was performed to examine associations between the KSHV serostatus and all of the questionnaire variables. Factors that were significant in univariate analyses at $\mathrm{P}=0.100$ were included in a multivariate logistic regression analysis, to identify which factors, if any, were independently associated with KSHV infection. Odds ratios (ORs) and 95\% confidence intervals (CIs) were used to quantify the relationships, while P-values were calculated to indicate the statistical significance. P-values $<0.050$ were considered significant. The chi-square test was also performed when appropriate to compare antibody titers between KSHV -infected individuals and KS patients.

\section{List of abbreviations}

KS: Kaposi sarcoma; KSHV: Kaposi's sarcoma-associated herpesvirus; HHV-8: Human herpesvirus 8; AIDS: acquired immunodeficiency syndrome; PEL : primary effusion lymphoma; MCD: multicentric Castleman's disease; OR: Odds ratios; 95\% Cl: 95\% confidence intervals; HBV: hepatitis B viruses; $\mathrm{HCV}$ : hepatitis C viruses; BMI: Body mass index; ELISA: enzyme-linked immunosorbent assay; RT: room temperature; ORF: open reading frame

\section{Acknowledgements}

This work was supported by the Youth Funds of Xinjiang Autonomous Region (grant number 2009211B17).

\section{Author details}

'The First Teaching Hospital of Xinjiang Medical University, 1 Liyu Shan Road, Urumqi, Xinjiang, People's Republic of China. ${ }^{2}$ Institut Pasteur of Shanghai, Chinese Academy of Sciences, Shanghai, People's Republic of China.

\section{Authors' contributions}

HW carried out study design, sample collection, and statistical analyses performance and participated in the antibody detection. JL and LL participated in sample collection. Dilimulati and ZH R wrote and collected the questionnaire. HW participated in the design of the study and carried out statistical analyses. XW conceived of the study, and participated in its design and coordination and helped to draft the manuscript. All authors read and approved the final manuscript.

\section{Competing interests}

The authors declare that they have no competing interests.

Received: 16 November 2010 Accepted: 14 December 2011 Published: 14 December 2011

\section{References}

1. Simonart Thierry: Role of environmental factors in the pathogenesis of classic and African-endemic Kaposi sarcoma. Cancer Letters 2006, 244:1-7.

2. Chang $Y$, Cesarman E, Pessin MS, et al: Identification of herpesvirus-like DNA sequences in AIDS-associated Kaposi's sarcoma. Science 1994, 266:1865-1869.

3. Schalling $M$, Ekman $M$, Kaaya EE, et al: A role for a new herpesvirus (KSHV) in different forms of Kaposi's sarcoma. Nat Med 1995, 1:707-708.

4. Hladik W, Dollard SC, Mermin J, et al: Transmission of human herpesvirus 8 by blood transfusion. N Engl J Med 2006, 355:1331-1338.

5. Beral V, Peterman TA, Berkelman RL, et al: Kaposi's sarcoma among persons with AIDS: a sexually transmitted infection? Lancet 1990, 335:123-128.

6. Giovanni S, Antonio S, Barbara I, et al: Sirolimus for Kaposi's Sarcoma in Renal-Transplant Recipients. N Engl J Med 2005, 352:1317-1323.

7. Gao SJ, Kingsley L, Li M, et al: KSHV antibodies among Americans, Italians and Ugandans with and without Kaposi's sarcoma. Nat Med 1996, 2:925-928.

8. Whitby D, Howard MR, Tenant-Flowers M, et al: Detection of Kaposi's sarcoma associated herpesvirus in peripheral blood of HIV-infected individuals and progression to Kaposi's sarcoma. Lancet 1995, 346:799-802.

9. Zavitsanou A, Malloiri M, Sypsa V, et al: Seroepidemiology of human herpesvirus 8 (HHV-8) infection in injecting drug users. Epidemiol Infect 2010, 138:403-408

10. Abel VB, Thomas FS.: Kaposi's Sarcoma-associated Herpesvirus(KSHV/ HHV8): Key Aspects of Epidemiology and Pathogenesis. AIDS Rev 2003, 5:222-229

11. Boshoff C, Schulz TF, Kennedy MM, et al: Kaposi's sarcoma-associated herpesvirus infects endothelial and spindle cells. Nat Med 1995, 1:1274-1278.

12. Staskus KA, Zhong W, Gebhard K, et al: Kaposi's sarcoma-associated herpesvirus gene expression in endothelial (spindle) tumor cells. J Virol 1997, 71:715-719.

13. Moore PS, Kingsley LA, Holmberg SD, et al: Kaposi's sarcoma-associated herpesviurs infection prior to onset of Kaposi's sarcoma. AIDS 1996, 10:175-180.

14. Kedes DH, Ganem D, Ameli N, et al: The prevalence of serum antibody to human herpesvirus 8 (Kaposi sarcoma-associated herpesvirus) among HIV-seropositive and high-risk HIV-seronegative women. JAMA 1997, 277:478-481.

15. Rezza G, Lennette ET, Giuliani M, et al: Prevalence and determinants of anti-lytic and anti-latent antibodies to human herpesvirus-8 among Italian individuals at risk of sexually and parenterally transmitted infections. Intl J Cancer 1998, 77:361-365.

16. Gao SJ, Kingsley L, Li M, et al: KSHV antibodies among Americans, Italians and Ugandans with and without Kaposi's sarcoma. Nat Med 1996, 2:925-928.

17. Boshoff C, Weiss RA: Epidemiology and pathogenesis of Kaposi's sarcoma-associated herpesvirus. Phil Trans R Soc Lon Biol Sci 2001, 356:517-534.

18. Fu B, Sun F, Li B, et al: Seroprevalence of Kaposi's Sarcoma-associated Herpesvirus and Risk Factors in Xinjiang, China. J Med Virol 2009, 81:1422-1431.

19. Fang Q, Liu J, Q BZ, et al: Seroprevalence of Kapsosi's sarcom-associated herpesvirus in the central population from Hubei provine. Viroligica Sinica 2006, 21:97-101

20. Wang $G Q, X u H$, Wang $Y K$, et al: Higher prevalence of human herpesvirus 8 DNA sequence and specific lgG antibodies in patients with pemphigus in China. J Am Acad Dermatol 2005, 52:460-467.

21. He F, Wang $X$, He B, et al: Human herpesvirus 8: serovprevalence and correlates in tumor patients from Xinjiang, China. J Med Virol 2007, 79:161-166.

22. Du W, Chen $G$, Sun $H$, et al: Antibody to human herpesvirus type 8 in the general populations of Xinjiang Autonomous Region. Chinese J Exp ClinVirol 2000, 14:44-47

23. Wang $X$, He B, Zhang ZX, et al: Human herpesvirus-8 in northwestern China: epidemiology and characterization among blood donors. Virology Journal 2010, 7:62-69. 
24. Sitas F, Carrara H, Beral V, et al: Antibodies against human herpesvirus 8 in black South African patients with cancer. N Engl J Med 1999, 340:1863-1871.

25. Baillargeon J, Deng JH, Hettler E, et al: Seroprevalence of Kaposi's sarcoma-associated herpesvirus infection among blood donors from Texas. Ann Epidemiol 2001, 11:512-518.

26. Sitas F, Newton R: Kaposi's sarcoma in South Africa. J Nati Cancer Inst Monogr 2001, 28:1-4.

27. Stein $L$, Carrara $H$, Norman $R$, et al: Antibodies against human herpesvirus 8 in South African renal transplant recipients and blood donors. Transplant Infection Disease 2004, 6:69-73.

28. Zavitsanou A, Sypsa V, Petrodaskalaki M, et al: Human herpesvirus 8 (HHV8) infection in healthy urban employees from Greece: Seroprevalence and associated factors. J Med Virol 2007, 79:591-596.

29. Zavitsanou A, Malliori M, Sypsa V, et al: Seroepidemiology of human herpesvirus 8 (HHV-8) infection in injecting drug users. Epidemiol Infect 2010, 138:403-408.

30. Li N, GUO SX, Zhang YH, et al: Investigation on knowledge, attitude and behaviour regarding hypertension among the Kazakh residents in Xinjiang. Journal of Shihezi University 2007, 25:203-206.

31. Angeloni A, Masala MV, Montesu MA, et al: Environmental factors influence the rate of human herpesvirus type 8 infection in a population with high incidence of classic Kaposi sarcoma. Clin Infect Dis 2006, 42 e66-e68.

32. Valdarchi C, Serraino D, Cordiali Fei $P$, et al: Demographic indicators and risk of infection with human herpesvirus type 8 in central Italy. Infection 2007, 35:22-25.

33. Pelser C, Vitale F, Whitby D, et al: Socio-Economic and Other Correlates of Kaposi Sarcoma-Associated Herpesvirus Seroprevalence Among Older Adults in Sicily. J Med Virol 2009, 81:1938-1944.

34. Wojcicki JM, Newton R, Urban Ml, et al: Risk factors for high anti-HHV-8 antibody titers ( $\geq 1: 51200)$ in black HIV-1 negative South African cancer patients: a case control study. BMC Infect Dis 2003, 3:21-32.

35. Mbulaiteye $S$, Atkinson J, Whitby D, et al: Risk factors for human herpesvirus 8 seropositivity in the AIDS Cancer Cohort Study. J Clin Virol 2006, 35:442-449.

doi:10.1186/1743-422X-8-539

Cite this article as: Wang et al: Seroprevalence and risk factors of Kaposi's sarcoma-associated herpesvirus infection among the general Uygur population from south and north region of Xinjiang, China. Virology Journal 2011 8:539.

\section{Submit your next manuscript to BioMed Central and take full advantage of:}

- Convenient online submission

- Thorough peer review

- No space constraints or color figure charges

- Immediate publication on acceptance

- Inclusion in PubMed, CAS, Scopus and Google Scholar

- Research which is freely available for redistribution 